FORM1. linterview women of reproductive age who are not currently pregnant or within 6 weeks of delivery, and desire delaying or limiting childbearing

Sequence number:

[Fill one number for each woman contacted in the order they were contacted at the health facility; if done over several days, continue unique sequence numbers]

\begin{tabular}{|c|c|c|}
\hline \multicolumn{3}{|l|}{ Identification of interview place } \\
\hline \multicolumn{3}{|l|}{ Province } \\
\hline \multicolumn{3}{|l|}{ CITY/MUNICIPALITY } \\
\hline \multicolumn{3}{|l|}{ BARANGAY } \\
\hline \multicolumn{3}{|l|}{ Health facility name } \\
\hline \multicolumn{3}{|l|}{ Home address (for home visit only) } \\
\hline \multicolumn{3}{|l|}{$\begin{array}{l}\text { Latitude and longitude } \\
\text { (Use the coordinate of GPS in a } \\
\text { mobile phone) }\end{array}$} \\
\hline \multicolumn{3}{|l|}{ Interview Record } \\
\hline \multicolumn{3}{|l|}{ Date of interview } \\
\hline \multicolumn{3}{|l|}{ Interviewer's name } \\
\hline $\begin{array}{l}\text { Health facility level where interview } \\
\text { took place }\end{array}$ & $\begin{array}{l}\text { 1. National hospital } \\
\text { 2. Regional hospital/Public medical center } \\
\text { 3. Provincial hospital } \\
\text { 4. District hospital } \\
\text { 5. Municipal hospital } \\
\text { 6. Rural health unit (RHU)/urban health } \\
\text { center(UHC)/Lying-in } \\
\text { 7. Barangay health station (BHS) } \\
\text { 8. Barangay supply/service point officer/BHW } \\
\text { 9. Mobile clinic } \\
\text { 10.Other (specify) }\end{array}$ & \\
\hline $\begin{array}{l}\text { Clinic where interview took place } \\
\text { (for large hospitals) }\end{array}$ & $\begin{array}{l}\text { 1. Reproductive health clinic for postnatal care } \\
\text { 2. Reproductive health clinic not related to } \\
\text { postnatal care } \\
\text { 3. Receiving vaccination or routine check-up for } \\
\text { child } \\
\text { 4. Seeking medical advice or treatment for } \\
\text { sickness or injury of child } \\
\text { 5. Seeking medical advice or treatment for } \\
\text { sickness or injury of herself } \\
\text { 6. Other (specify) }\end{array}$ & \\
\hline
\end{tabular}


Sequence Number:

[Write the same sequence number from Cover Page]

Instructions:

Read the information sheet. Answer questions. If the woman agrees to participate give the certificate of consent for her to sign.

Then start the Screening Form.

\section{Screening Form}

State: "We would like to start by asking a few questions that determine if you are eligible for the survey." "In kami ini mabayah mangasubu maniyu tiyu kaniyo bang in kamo manjari para ha survey namo"

\begin{tabular}{|c|c|c|c|}
\hline 001 & $\begin{array}{l}\text { How old were you on your last } \\
\text { birthday? } \\
\text { "Pila tahun na kaw amun last } \\
\text { birth day mo?" }\end{array}$ & Age in completed years & $\begin{array}{l}18-49 \text { years }->002 \\
\text { Other }->009\end{array}$ \\
\hline 002 & $\begin{array}{l}\text { Are you pregnant now? } \\
\text { "Burus kaw bihaun?" }\end{array}$ & $\begin{array}{l}\text { 1. Yes "Huun,Oo" } \\
\text { 2. No "Bukon" } \\
\text { 3. Unsure "Di ko } \\
\text { kaingatan" }\end{array}$ & $\begin{array}{l}1->009 \\
2->003 \\
3->003\end{array}$ \\
\hline 003 & $\begin{array}{l}\text { What is the name of your last } \\
\text { baby? } \\
\text { "Uno in ngan sin kamanghuran } \\
\text { anak mo?" } \\
\text { Record name }\end{array}$ & $\begin{array}{l}\text { 1. Name: } \\
\text { 2. No previous baby }\end{array}$ & $\begin{array}{l}1->004 \\
2->006\end{array}$ \\
\hline 004 & $\begin{array}{l}\text { In what month and year was } \\
\text { NAME born? } \\
\text { "Uno bulan iban tahun sya } \\
\text { piyanganak?" } \\
\text { (probe: when is his or her } \\
\text { birthday) }\end{array}$ & $\begin{array}{l}\text { Month: }-- \\
\text { Year:__ _ _ - }\end{array}$ & $\begin{array}{l}\text { Age } \geq 6 \text { wks }->005 \\
\text { Age }<6 \text { wks }->009\end{array}$ \\
\hline 005 & $\begin{array}{l}\text { Has your menstrual period } \\
\text { returned since the birth of } \\
\text { NAME? } \\
\text { "Nagbalik na ba in pagdugu mo } \\
\text { amun pagubus sin pag anak mo?" }\end{array}$ & $\begin{array}{l}\text { 1. Yes } \\
\text { 2. No }\end{array}$ & $\begin{array}{l}1->006 \\
2->006\end{array}$ \\
\hline 006 & $\begin{array}{l}\text { Now I have some questions about } \\
\text { the future. Would you like to } \\
\text { have (a/another) child, or would } \\
\text { you prefer not to have any (more) } \\
\text { children? } \\
\text { "Bihaun mangasubu ako. } \\
\text { Mabayah pa kaw manganak } \\
\text { magbalik atawa dih na?" }\end{array}$ & $\begin{array}{l}\text { 1. Have (a/another ) child } \\
\text { "Magkaanak magbalik" } \\
\text { 2. No more/none "Dih } \\
\text { na" } \\
\text { 3. Cannot get pregnant } \\
\text { "Dih na maburus" } \\
\text { 4. Undecided / don't } \\
\text { know "Dih ku } \\
\text { kaingatan" }\end{array}$ & $\begin{array}{l}1->007 \\
2->008 \\
3->009 \\
4->009\end{array}$ \\
\hline 007 & $\begin{array}{l}\text { Do you want (a/another) child } \\
\text { soon? } \\
\text { "Mabayah pa kaw magka anak } \\
\text { magbalik ha susungan?" }\end{array}$ & $\begin{array}{l}\text { 1. Yes "Huun,Oo" } \\
\text { 2. No, want to wait "Dih } \\
\text { muna" } \\
\text { 3. Don't know "Inday ku" }\end{array}$ & $\begin{array}{l}1->009 \\
2->008 \\
3->009\end{array}$ \\
\hline 008 & $\begin{array}{l}\text { Are you or your husband/partner } \\
\text { currently doing something or } \\
\text { using any method to delay or } \\
\text { avoid getting pregnant? } \\
\text { "Awun iyuusal nyo iban bana mo } \\
\text { or hinang nyo para kaw di } \\
\text { maburus magtuy" }\end{array}$ & $\begin{array}{l}\text { 1. Yes } \\
\text { 2. No }\end{array}$ & $\begin{array}{l}1->101 \\
2->101 \\
\text { To achieve a total } \\
\text { of } 5 \text { users and } \\
\text { non-users } \\
\text { (hospitals) and } 3 \\
\text { users and } 3 \text { non- } \\
\text { users (health } \\
\text { centres). }\end{array}$ \\
\hline 009 & \multicolumn{3}{|c|}{$\begin{array}{l}\text { Thank the woman, indicate ineligibility for the survey and stop the interview. Enter this } \\
\text { woman into "number of women contacted". Then find another woman to interview. }\end{array}$} \\
\hline
\end{tabular}


FORM1. Interview of women of reproductive age who are not currently pregnant or within 6 weeks of delivery, and desire delaying or limiting childbearing

Sequence Number:

[Write the same sequence number from Cover Page]

\begin{tabular}{|c|c|c|c|}
\hline NO. & \multicolumn{3}{|l|}{ Section 1. Respondent background } \\
\hline 101 & $\begin{array}{l}\text { In (month of interview) } 2016 \text {, } \\
\text { did you live in a city, in a town } \\
\text { proper/ poblacion, in the barrio } \\
\text { or rural area, or abroad? } \\
\text { "Ha tahun } 2016 \text { nakabutang } \\
\text { kaw ha city proper, barangay } \\
\text { atawa abroad?" }\end{array}$ & $\begin{array}{l}\text { 1. City } \\
\text { 2. TOWN PROPERIPOBLACION } \\
\text { 3. BARRIO/RURAL AREA } \\
\text { 4. ABROAD } \\
\text { 5. DON'T KNOW }\end{array}$ & $->102$ \\
\hline 102 & $\begin{array}{l}\text { What is your marital status } \\
\text { now? } \\
\text { "In ikaw yan budjang, awun na } \\
\text { bana (natiyaun na), nagbugit } \\
\text { iban bana? }\end{array}$ & $\begin{array}{l}\text { 1. Never married or never lived } \\
\text { with a man "budjang" } \\
\text { 2. Currently married "awun } \\
\text { bana (natiyaun na)" } \\
\text { 3. Currently living with a man } \\
\text { "not applicable for Islam" } \\
\text { 4. Divorced/separated/widow } \\
\text { and not currently living with } \\
\text { a man "nagbugit iban bana" }\end{array}$ & $->103$ \\
\hline 103 & $\begin{array}{l}\text { What is your highest level of } \\
\text { education attended, whether or } \\
\text { not that level was completed? } \\
\text { "Uno in natalus mo ha iskul?" }\end{array}$ & $\begin{array}{l}\text { 1. No education "uway naka } \\
\text { iskul" } \\
\text { 2. Elementary } \\
\text { 3. High school } \\
\text { 4. College } \\
\text { 5. Post-graduate } \\
\end{array}$ & $->104$ \\
\hline 104 & $\begin{array}{l}\text { How many children do you have } \\
\text { who are still alive? } \\
\text { "Pila in anak mo bihaun?" }\end{array}$ & Number of children alive & $->106$ \\
\hline 105 & $\begin{array}{l}\text { Did you or someone else do } \\
\text { anything to end any of your past } \\
\text { pregnancies? } \\
\text { "Bakas awun nahinang mo para } \\
\text { di matuy in pagburus?" }\end{array}$ & $\begin{array}{l}\text { 1. Yes } \\
\text { 2. No }\end{array}$ & $\begin{array}{l}1->107 \\
2->108\end{array}$ \\
\hline 106 & $\begin{array}{l}\text { How many pregnancies did you } \\
\text { or someone else do anything to } \\
\text { end? } \\
\text { "Nakapila nahinang?" }\end{array}$ & Number of induced abortion & $->108$ \\
\hline 107 & $\begin{array}{l}\text { Are you covered by any health } \\
\text { insurance, either as member or } \\
\text { dependent? } \\
\text { "Awun health insurance nyo?" }\end{array}$ & $\begin{array}{l}\text { 1. Not covered } \\
\text { 2. Philhealth } \\
\text { 3. Government Service } \\
\text { Insurance System } \\
\text { 4. Social Security System } \\
\text { 5. Private insurance } \\
\text { company/Health } \\
\text { maintenance organization } \\
\text { /Pre-need insurance plan } \\
\text { company } \\
\text { 6. Other (Specify) }\end{array}$ & $->201$ \\
\hline
\end{tabular}

\begin{tabular}{|l|l|l|l|l|}
\hline NO. & Section 2. Current use of FP & & $1->202$ \\
\hline 201 & $\begin{array}{l}\text { REVIEW: Are you or your } \\
\text { husband/partner currently } \\
\text { doing something or using any } \\
\text { method to delay or avoid } \\
\text { getting pregnant? }\end{array}$ & $\begin{array}{l}\text { 4. No } \\
\text { 4. Yes }\end{array}$ & & \\
\hline
\end{tabular}




\begin{tabular}{|c|c|c|c|c|c|c|c|}
\hline & $\begin{array}{l}\text { "Awun iyuusal nyo iban bana } \\
\text { mo or hinang nyo para kaw di } \\
\text { maburus magtuy" }\end{array}$ & & & & & & \\
\hline 202 & $\begin{array}{l}\text { Which method are you } \\
\text { currently using? } \\
\text { "Uno in mga hinang nyo?" } \\
\text { WRITE DOWN ALL MENTIONED. }\end{array}$ & \multicolumn{4}{|c|}{$\begin{array}{ll}\text { 1. } & \text { Female sterilization } \\
\text { 2. } & \text { Male sterilization } \\
\text { 3. } & \text { IUD } \\
\text { 4. } & \text { Injectable (e.g.DMPA) } \\
\text { 5. Implants } \\
\text { 6. } & \text { Patch } \\
\text { 7. } & \text { Pill } \\
\text { 8. } & \text { Condom } \\
\text { 9. } & \text { Female condom } \\
\text { 10. } & \text { Diaphragm } \\
\text { 11. } & \text { Form/Jelly/Cream } \\
\text { 12. } & \text { Mucus/Billings/Ovulation } \\
\text { 13. } & \text { Basal body temperature } \\
\text { 14. Symptothermal } \\
\text { 15. Standard days method } \\
\text { 16. LAM } \\
\text { 17. Calendar/Rhythm/Periodic } \\
\text { abstinence } \\
\text { 18. } \text { Withdrawal } \\
\text { 19. Other traditional method } \\
\text { 20. Other modern method } \\
\text { (specify) }\end{array}$} & & \multirow[t]{2}{*}{$->203$} \\
\hline & LINE NUMBER & 01 & 02 & 03 & 04 & 05 & \\
\hline 203 & $\begin{array}{l}\text { Now I would like to ask you one } \\
\text { by one about all methods you } \\
\text { are using now. } \\
\text { "Bihaun mabyah ako } \\
\text { mangasubu knyo pasal ha mga } \\
\text { method nyo" } \\
\text { RECORD ALL METHODS BEING } \\
\text { USED NOW, ONE METHOD PER } \\
\text { ONE LINE NUMBER. } \\
\text { IF THERE ARE MORE THAN } 5 \\
\text { METHODS, USE ADDITIONAL } \\
\text { QUESTIONNAIRE. } \\
\text { 1. Female sterilization } \\
\text { 2. Male sterilization } \\
\text { 3. IUD } \\
\text { 4. Injectable (e.g.DMPA) } \\
\text { 5. Implants } \\
\text { 6. Patch } \\
\text { 7. Pill } \\
\text { 8. Condom } \\
\text { 9. Female condom } \\
\text { 10. Diaphragm } \\
\text { 11. Form/Jelly/Cream } \\
\text { 12. Mucus/Billings/Ovulation } \\
\text { 13. Basal body temperature } \\
\text { 14. Symptothermal } \\
\text { 15. Standard days method } \\
\text { 16. LAM } \\
\text { 17. Calendar/Rhythm/Periodic } \\
\text { 18. Withdrawal } \\
\text { 19. Other traditional method } \\
\text { 20. Other modern method } \\
\text { (specify) }\end{array}$ & & & & & & $->204$ \\
\hline
\end{tabular}




\begin{tabular}{|c|c|c|c|}
\hline 204 & $\begin{array}{l}\text { Where did you obtain that } \\
\text { method when you first started } \\
\text { using it? } \\
\text { "Hawnu mo nakawa in mga } \\
\text { method ini?" } \\
\text { 1. National hospital } \\
\text { 2. Regional hospital/Public } \\
\text { medical center } \\
\text { 3. Provincial hospital } \\
\text { 4. District hospital } \\
\text { 5. Municipal hospital } \\
\text { 6. Rural health unit } \\
\text { (RHU)/urban health } \\
\text { center(UHC)/Lying-in } \\
\text { 7. Barangay health station } \\
\text { (BHS) } \\
\text { 8. Barangay supply/service } \\
\text { point officer/BHW } \\
\text { 9. Mobile clinic } \\
\text { 10.Other (specify. Private } \\
\text { facility is included here) }\end{array}$ & & $->205$ \\
\hline 205 & $\begin{array}{l}\text { What was the purpose of your } \\
\text { going to the health facility on } \\
\text { the day you first received the } \\
\text { contraceptive method? } \\
\text { "Uno in natalih mo amun } \\
\text { miyadtu kaw pa health facility } \\
\text { ubus dinihilan kaw } \\
\text { contraceptive method?" } \\
\text { 1. Prenatal care } \\
\text { 2. Giving birth, while a women } \\
\text { is still in the facility } \\
\text { 3. Reproductive health } \\
\text { outpatient clinic for } \\
\text { postnatal care } \\
\text { 4. Reproductive health clinic } \\
\text { not related to postnatal care } \\
\text { 5. Receiving vaccination or } \\
\text { routine check up for child } \\
\text { 6. Seeking medical advice or } \\
\text { treatment for sickness or } \\
\text { injury of child } \\
\text { 7. Seeking medical advice or } \\
\text { treatment for sickness or } \\
\text { injury of herself } \\
\text { 8. Adolescent clinic } \\
\text { 9. Other (specify) }\end{array}$ & & $\begin{array}{l}->203 . \\
\text { Repeat until } \\
\text { all methods } \\
\text { were } \\
\text { explained. } \\
\text { Then ->206 }\end{array}$ \\
\hline 206 & $\begin{array}{l}\text { If you are not using any method } \\
\text { to delay or avoid getting } \\
\text { pregnant now, have you or your } \\
\text { sexual partner done something } \\
\text { or used a method to delay or } \\
\text { avoid getting pregnant in the } \\
\text { past? } \\
\text { "Bang kaw y nagusal protection } \\
\text { para dih maburus bihaun. } \\
\text { Nakausal kamo iban banah mo } \\
\text { sin tagna?" }\end{array}$ & $\begin{array}{ll}\text { 1. Yes } \\
\text { 2. No }\end{array}$ & $\begin{array}{l}1->207 \\
2->301\end{array}$ \\
\hline
\end{tabular}




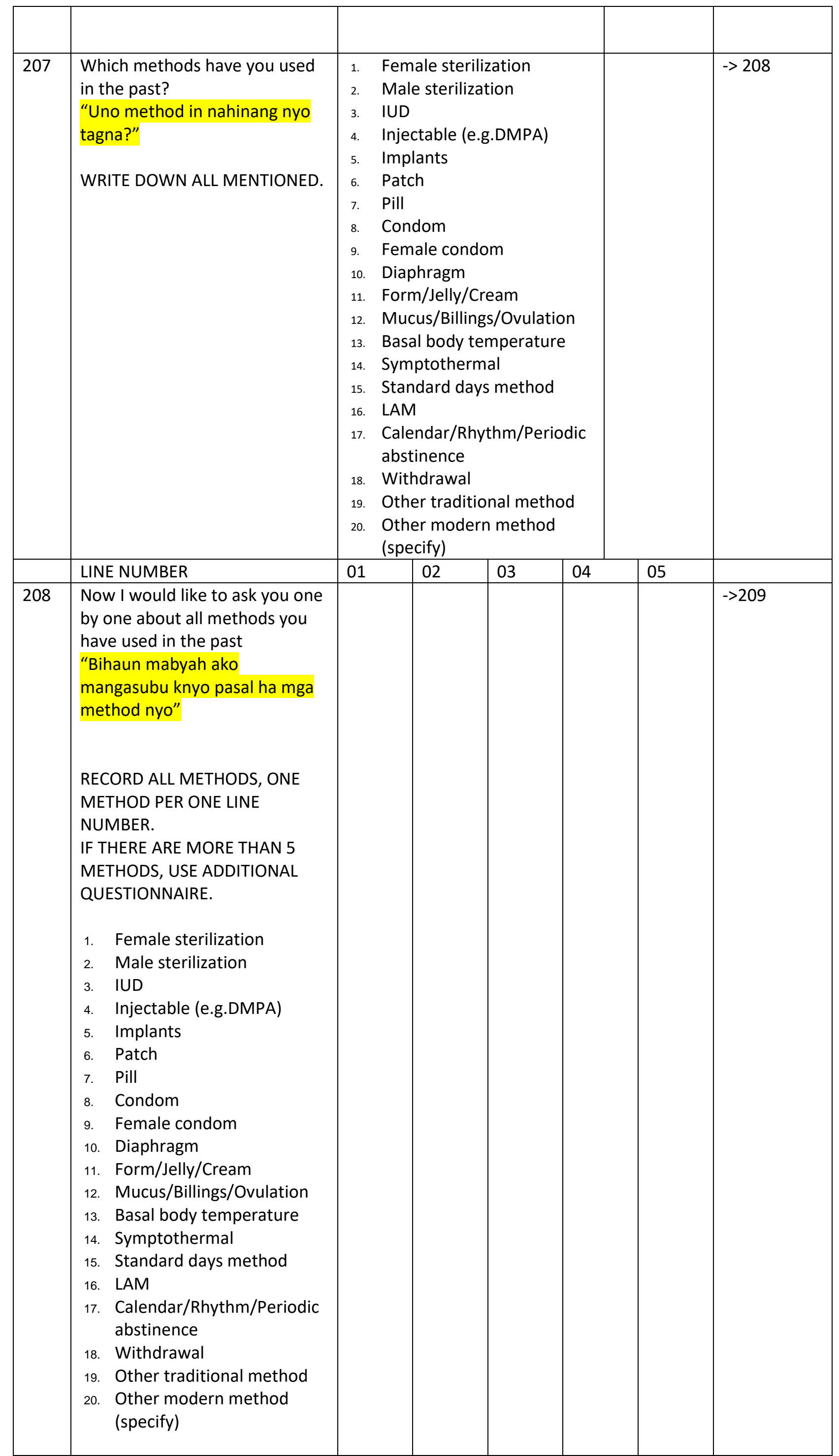




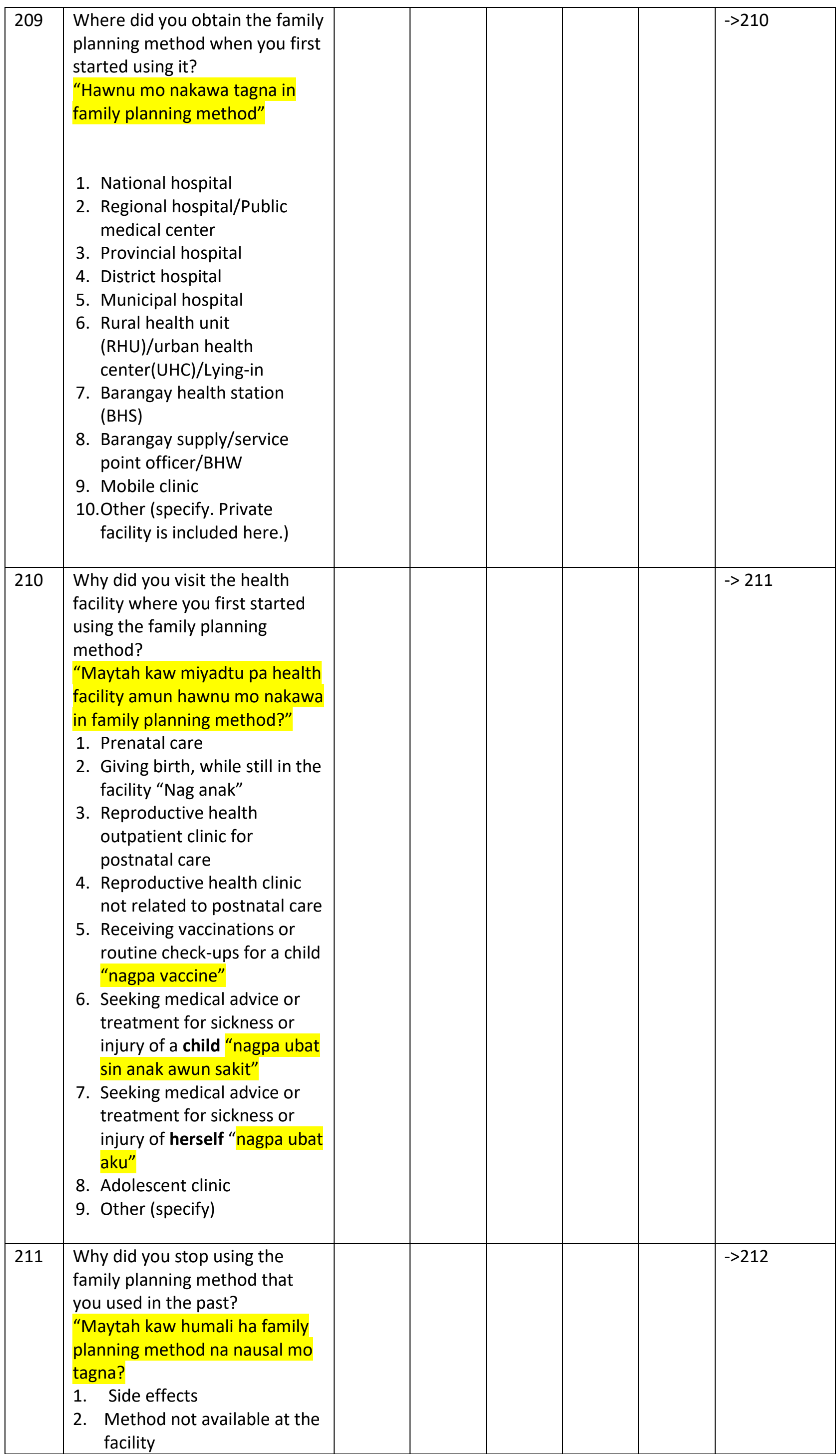


3. Concerns about risks of pregnancy

4. Could not afford to purchase

5. Health worker did not continue to provide the method

6. Advice of friends, relatives, neighbors

7. Husband/partner did not support

8. Wanted to get pregnant

9. Other (specify): 


\begin{tabular}{|c|c|c|c|c|c|c|c|c|}
\hline \multirow[b]{2}{*}{301} & \multicolumn{8}{|c|}{ Section 3. FP Concerns and Today's FP counseling } \\
\hline & $\begin{array}{l}\text { Do you have any health } \\
\text { concerns about any type of } \\
\text { family planning method? } \\
\text { "Awun mga pangasubu mo } \\
\text { pasal ha family planning } \\
\text { method?" }\end{array}$ & & $\begin{array}{l}\text { Yes } \\
\text { No }\end{array}$ & & & & & $\begin{array}{l}1->302 \\
2->305\end{array}$ \\
\hline & LINE NUMBER & 01 & 02 & 03 & 04 & 05 & 06 & \\
\hline 302 & $\begin{array}{l}\text { What are your health concerns } \\
\text { about family planning methods? } \\
\text { Please tell me one by one. } \\
\text { "Uno in mga pangasubo mo } \\
\text { pasal ha family planning } \\
\text { methods?" Iyana kako. } \\
\text { USE ONE LINE NUMBER FOR } \\
\text { ONE CONCERN. WRITE DOWN } \\
\text { ALL MENTIONED CONCERNS. } \\
\text { IF THERE ARE MORE THAN } 6 \\
\text { CONCERNS, USE ADDITIONAL } \\
\text { QUESTIONNAIRE. } \\
\text { 1. Cause cancer in the uterus } \\
\text { 2. Cause cysts in the uterus } \\
\text { 3. Cause infection of the } \\
\text { uterus } \\
\text { 4. Cause frequent bleeding } \\
\text { 5. Cause thyroid problems } \\
\text { 6. Cause/worse asthma } \\
\text { 7. Cause/worse lots of veins } \\
\text { 8. Cause dry skin, skin } \\
\text { disease } \\
\text { 9. Cause edema } \\
\text { 10.Cause weight gain } \\
\text { 11.Cause weight loss } \\
\text { 12.Cause bloated stomach } \\
\text { 13.Cause headache } \\
\text { 14.Cause irritability } \\
\text { 15.Increase libido/turn into a } \\
\text { maniac } \\
\text { 16.Cause loss/reduce of libido } \\
\text { 17.Cause loss/reduce of } \\
\text { sexual satisfaction } \\
\text { 18.One will not have children } \\
\text { anymore } \\
\text { 19.Not fully effective, woman } \\
\text { could still get pregnant } \\
\text { 20.When it does not work, } \\
\text { the baby is born with } \\
\text { abnormalities } \\
\text { 21. Results in mortal sin } \\
\text { because it is against church } \\
\text { teachings } \\
\text { inside the body and } \\
\text { foctors will not be able to } \\
\text { IUD/Implants } \\
\text { 22.Melt or move aroushshed out } \\
\text { find }\end{array}$ & & & & & & & $->303$ \\
\hline
\end{tabular}




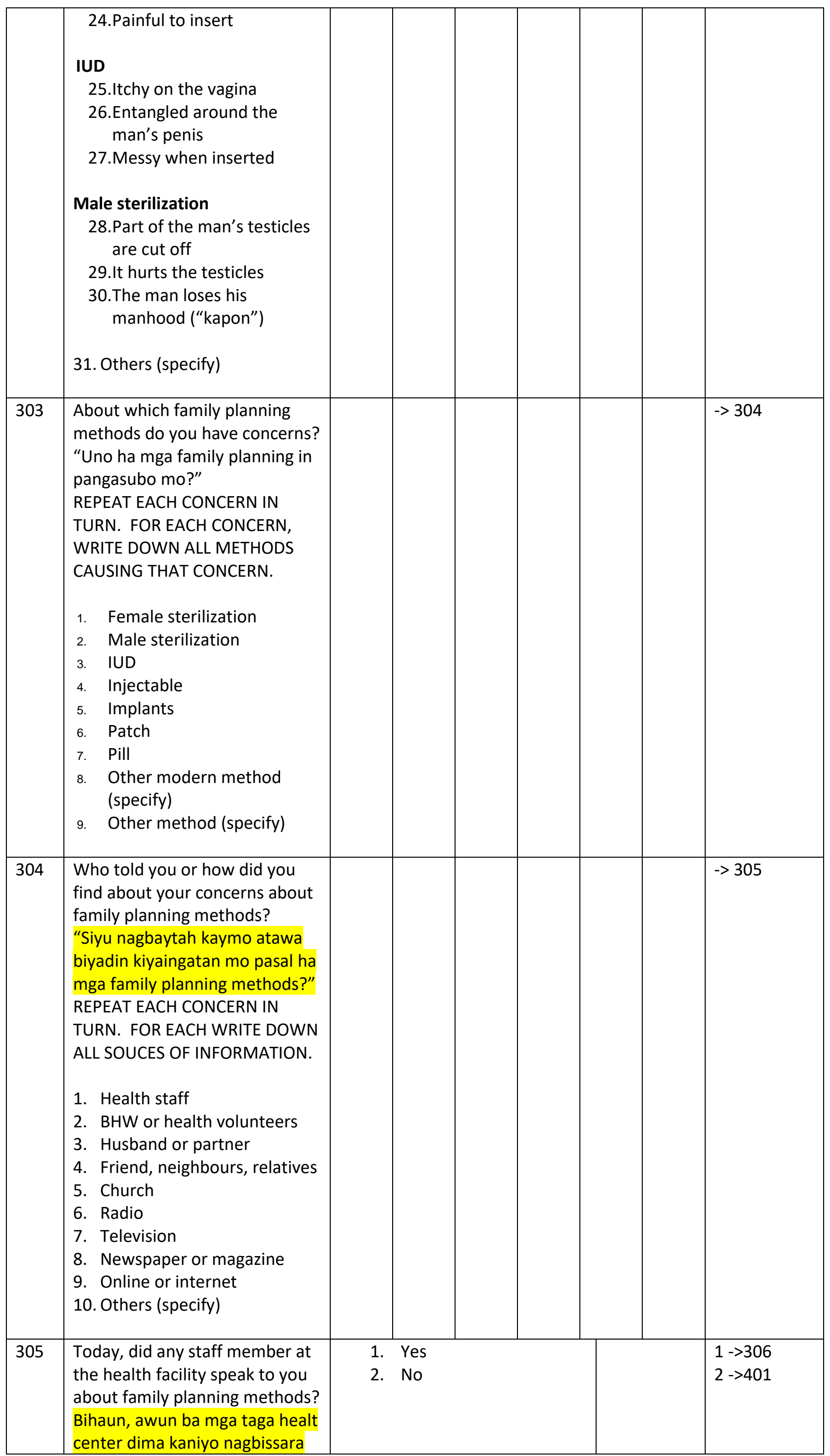




\begin{tabular}{|c|c|c|c|}
\hline & $\begin{array}{l}\text { pasal ha family planning } \\
\text { method?" }\end{array}$ & & \\
\hline 306 & $\begin{array}{l}\text { Did the health worker ask you } \\
\text { about your concerns? } \\
\text { "Iyasubo kaw sin tau ha health } \\
\text { worker pasal ha mga problema } \\
\text { (concern) nyo?" }\end{array}$ & $\begin{array}{l}\text { 1. Yes } \\
\text { 2. No }\end{array}$ & $\begin{array}{l}1->307 \\
2->309\end{array}$ \\
\hline 307 & $\begin{array}{l}\text { Do you feel the health worker } \\
\text { understands your concerns? } \\
\text { "Kiyahatihan da kaw sin health } \\
\text { worker pasal sin problema } \\
\text { (concern) mo?" }\end{array}$ & $\begin{array}{l}\text { 1. Yes } \\
\text { 2. No }\end{array}$ & $->308$ \\
\hline 308 & $\begin{array}{l}\text { Did the health worker help you } \\
\text { to find solutions to your } \\
\text { concerns? } \\
\text { Timabang ba in health worker } \\
\text { kymo pasal ha problema } \\
\text { (concern)?" }\end{array}$ & $\begin{array}{l}\text { 1. Yes } \\
\text { 2. No }\end{array}$ & $->309$ \\
\hline 309 & $\begin{array}{l}\text { Did the health worker offer you } \\
\text { information how different } \\
\text { family planning methods work? } \\
\text { "Nagdihil ba in health worker } \\
\text { bang uno-uno in karayawan sin } \\
\text { mga family planning methods?" }\end{array}$ & $\begin{array}{l}\text { 1. Yes } \\
\text { 2. No }\end{array}$ & $\begin{array}{l}1->310 \\
2->312\end{array}$ \\
\hline 310 & $\begin{array}{l}\text { Which methods did health } \\
\text { worker mention today? } \\
\text { "Uno method in biyaytah knyo } \\
\text { adlaw ini?" }\end{array}$ & $\begin{array}{ll}\text { 1. } & \text { Female sterilization } \\
\text { 2. } & \text { Male sterilization } \\
\text { 3. IUD } \\
\text { 4. Injectable (e.g.DMPA) } \\
\text { 5. Implants } \\
\text { 6. Patch } \\
\text { 7. Pill } \\
\text { 8. Condom } \\
\text { 9. Female condom } \\
\text { 10. Diaphragm } \\
\text { 11. Form/Jelly/Cream } \\
\text { 12. Mucus/Billings/Ovulation } \\
\text { 13. Basal body temperature } \\
\text { 14. Symptothermal } \\
\text { 15. Standard days method } \\
\text { 16. LAM } \\
\text { 17. Calendar/Rhythm/Periodic } \\
\text { abstinence } \\
\text { 18. Withdrawal } \\
\text { 19. Other traditional method } \\
\text { 20. Other modern method } \\
\text { (specify) }\end{array}$ & $->311$ \\
\hline 311 & $\begin{array}{l}\text { Did the health worker tell you } \\
\text { about side-effects or problems } \\
\text { you might have with any } \\
\text { methods of family planning? } \\
\text { "Kiya baytaan kamo sin health } \\
\text { worker pasal sin problema sin } \\
\text { methods of family planning?" }\end{array}$ & $\begin{array}{l}\text { 1. Yes } \\
\text { 2. No }\end{array}$ & $->312$ \\
\hline 312 & $\begin{array}{l}\text { Did the health worker offer you } \\
\text { information how your family } \\
\text { planning method works? } \\
\text { "Kiyabaytaan ba kaw sin health } \\
\text { worker bang uno in mahinang } \\
\text { sin family planning methods?" }\end{array}$ & $\begin{array}{ll}\text { 1. } & \text { Yes } \\
\text { 2. } & \text { No } \\
\text { 3. } & \text { N/A (not using a } \\
& \text { method now) }\end{array}$ & $\begin{array}{l}1->313 \\
2->313 \\
3->315\end{array}$ \\
\hline
\end{tabular}




\begin{tabular}{|c|c|c|c|}
\hline 313 & $\begin{array}{l}\text { Did the health worker explain } \\
\text { about the side effects of your } \\
\text { current method? } \\
\text { "Biyaytaan kaw sin healt worker } \\
\text { bng uno in side effect sin } \\
\text { iyuusal mo method bihaun?" }\end{array}$ & $\begin{array}{ll}\text { 1. } & \text { Yes } \\
\text { 2. No }\end{array}$ & -> 314 \\
\hline 314 & $\begin{array}{l}\text { Did the health worker ask you } \\
\text { to describe how you use your } \\
\text { current method? } \\
\text { "Iyasubo kaw sin health worker } \\
\text { bng bihadiin usalun in method } \\
\text { iyuusal mo?" }\end{array}$ & $\begin{array}{ll}\text { 1. } & \text { Yes } \\
\text { 2. No }\end{array}$ & $->401$ \\
\hline 315 & $\begin{array}{l}\text { After receiving FP counselling } \\
\text { will you begin using a family } \\
\text { planning method today? } \\
\text { "Bihaun kaingatan mo na in } \\
\text { pasal ha family planning, usalun } \\
\text { mo na bihaun in family } \\
\text { planning?" }\end{array}$ & $\begin{array}{ll}\text { 1. Yes } \\
\text { 2. No }\end{array}$ & $\begin{array}{l}1 \text {-> } 317 \\
2->316\end{array}$ \\
\hline 316 & $\begin{array}{l}\text { After receiving FP counselling } \\
\text { will you begin using, do you } \\
\text { think you will use a } \\
\text { contraceptive method anytime } \\
\text { in the future? } \\
\text { "Bihaun kaingatan mo na in } \\
\text { pasal ha family planning atawa } \\
\text { ha susungan?" }\end{array}$ & $\begin{array}{ll}\text { 1. Yes } \\
\text { 2. No }\end{array}$ & $\begin{array}{l}1->317 \\
2->401\end{array}$ \\
\hline 317 & $\begin{array}{l}\text { Which contraceptive method } \\
\text { would you prefer to use? } \\
\text { "Uno contraceptive method in } \\
\text { kabayaan mo usalun?" }\end{array}$ & $\begin{array}{ll}\text { 1. } & \text { Female sterilization } \\
\text { 2. } & \text { Male sterilization } \\
\text { 3. IUD } \\
\text { 4. Injectable (e.g.DMPA) } \\
\text { 5. Implants } \\
\text { 6. } & \text { Patch } \\
\text { 7. } & \text { Pill } \\
\text { 8. } & \text { Condom } \\
\text { 9. } & \text { Female condom } \\
\text { 10. } & \text { Diaphragm } \\
\text { 11. } & \text { Form/Jelly/Cream } \\
\text { 12. } & \text { Mucus/Billings/Ovulation } \\
\text { 13. Basal body temperature } \\
\text { 14. Symptothermal } \\
\text { 15. Standard days method } \\
\text { 16. LAM } \\
\text { 17. Calendar/Rhythm/Periodic } \\
\text { abstinence } \\
\text { 18. Withdrawal } \\
\text { 19. Other traditional method } \\
\text { 20. Other modern method } \\
\text { (specify) }\end{array}$ & $->401$ \\
\hline
\end{tabular}




\begin{tabular}{|c|c|c|c|c|c|c|c|c|}
\hline \multirow{3}{*}{401} & \multicolumn{8}{|c|}{$\begin{array}{l}\text { Section } 4 \text {. Past Health facility visit and FP counseling } \\
\text { Do not count today's visit. }\end{array}$} \\
\hline & $\begin{array}{l}\text { Not including today, in the last } \\
12 \text { months, have you visited a } \\
\text { health facility for care for } \\
\text { yourself or your children for any } \\
\text { purpose? } \\
\text { "Uway iyagad in adlaw ini, } \\
\text { nakakadtu kaw pa health center } \\
\text { tagna amun last } 12 \text { months pa } \\
\text { healt facility atawa mga anak } \\
\text { mo?" }\end{array}$ & & $\begin{array}{l}\text { Yes } \\
\text { No }\end{array}$ & & & & & $\begin{array}{l}1->402 \\
2->\text { End of } \\
\text { interview }\end{array}$ \\
\hline & LINE NUMBER & 01 & 02 & 03 & 04 & 05 & 06 & \\
\hline 402 & $\begin{array}{l}\text { Now I would like to record all } \\
\text { your facility visits for last } 12 \\
\text { months. Start with the latest } \\
\text { visit you had. } \\
\text { Why did you visit a health } \\
\text { facility? } \\
\text { "Bihaun ilista ku in mga } \\
\text { pagkadtu mo pa health center } \\
\text { amun nakalabay } 12 \text { months. } \\
\text { Maytah kaw miyadtu pa health } \\
\text { facility?" } \\
\text { AFTER WRITING THE FIRST VISIT } \\
\text { IN LINE NUMBER 01, ASK Q403- } \\
\text { 410 FOR THAT VISIT. THEN ASK } \\
\text { THE 2nd LATEST VISIT TO WRITE } \\
\text { IN } 402 \text { LINE NUMBER 02, THEN } \\
\text { ASK Q } 403 \text { AND Q404. } \\
\text { REPEAT FOR ALL HEALTH } \\
\text { FACILITY VISITS FOR LAST 12 } \\
\text { MONTHS. } \\
\text { IF THERE ARE MORE THAN 6, } \\
\text { USE AN ADDITIONAL } \\
\text { QUESTIONNAIRE. } \\
\text { 1. Prenatal care } \\
\text { 2. Giving birth, while a women } \\
\text { is still in the facility } \\
\text { 3. Reproductive health } \\
\text { outpatient clinic for } \\
\text { postnatal care } \\
\text { 4. Reproductive health clinic } \\
\text { not related to postnatal care } \\
\text { 5. Receiving vaccination or } \\
\text { routine check up for child } \\
\text { 6. Seeking medical advice or } \\
\text { treatment for sickness or } \\
\text { injury of child } \\
\text { 7. Seeking medical advice or } \\
\text { treatment for sickness or } \\
\text { injury of herself } \\
\text { 8. Adolescent clinic } \\
\text { 9. Other (specify) } \\
\text { (n) }\end{array}$ & & & & & & & $->403$ \\
\hline 403 & $\begin{array}{l}\text { Where did you visit? } \\
\text { "Uno in kiyadtuhan mo?" } \\
\text { 1. National hospital } \\
\text { 2. Regional hospital/Public } \\
\text { medical center } \\
\text { 3. Provincial hospital }\end{array}$ & & & & & & & $->404$ \\
\hline
\end{tabular}




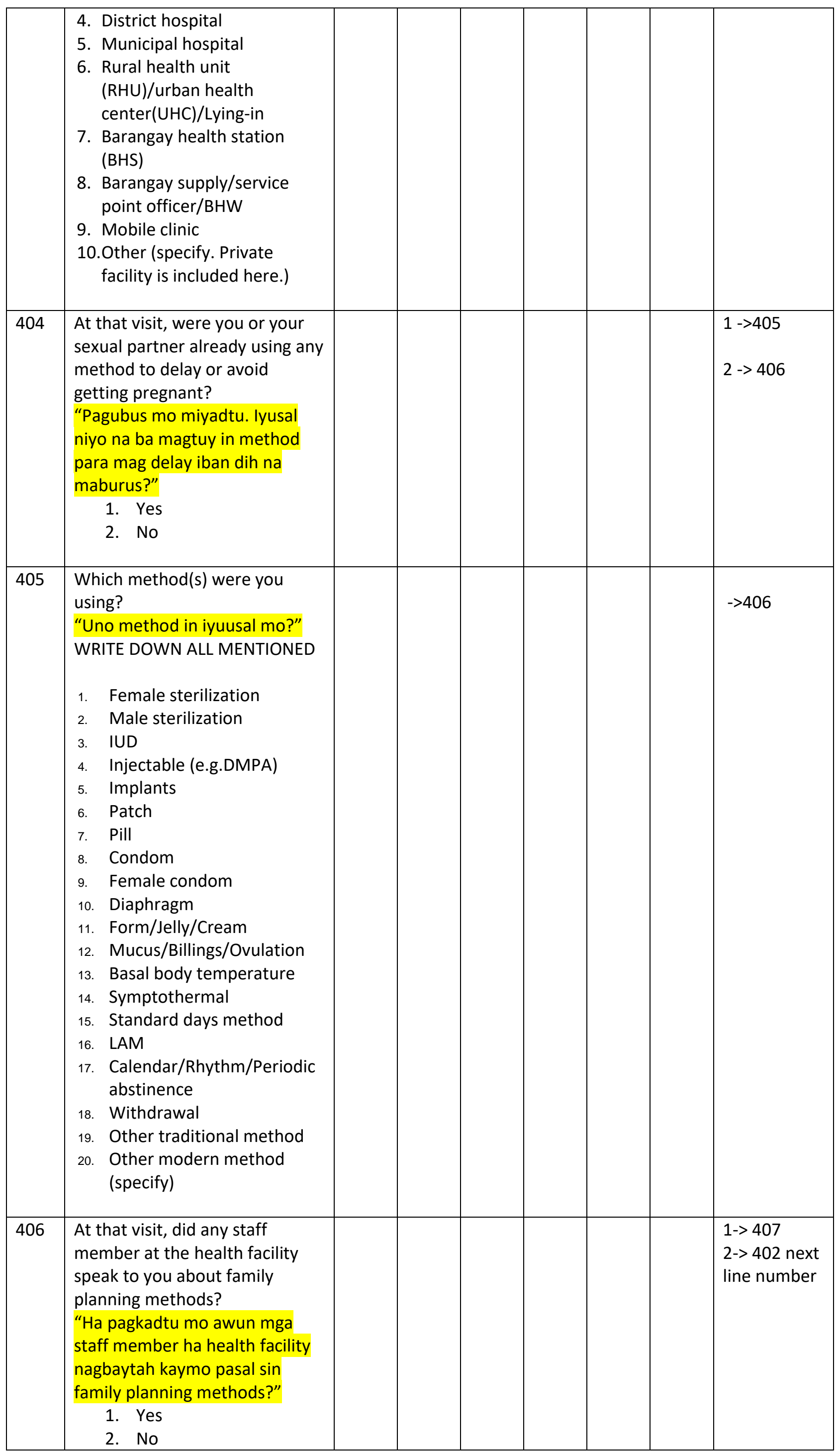




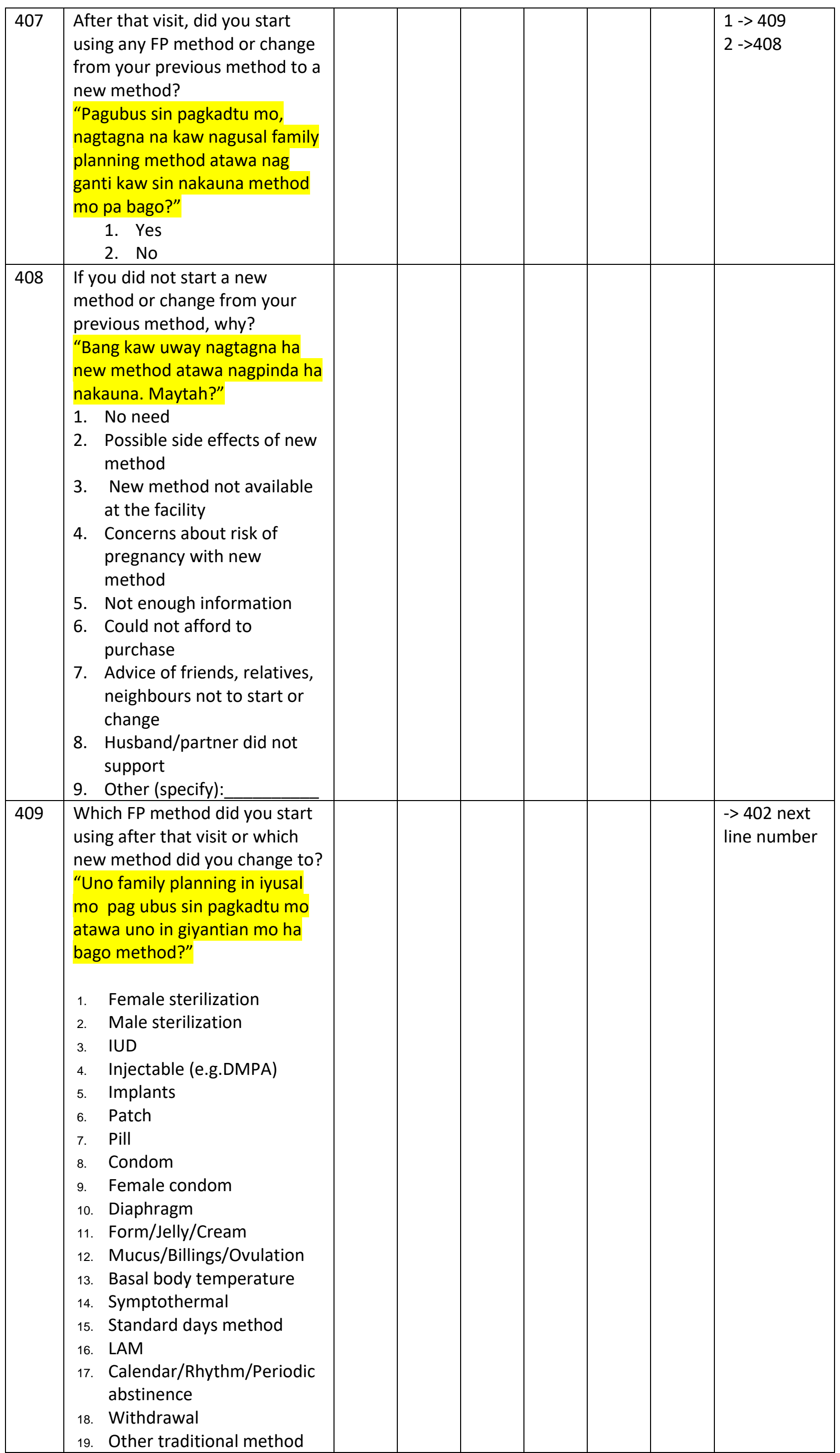




\section{END OF THE INTERVIEW}

